\title{
Corneal cross-linking methods and outcomes: A review
}

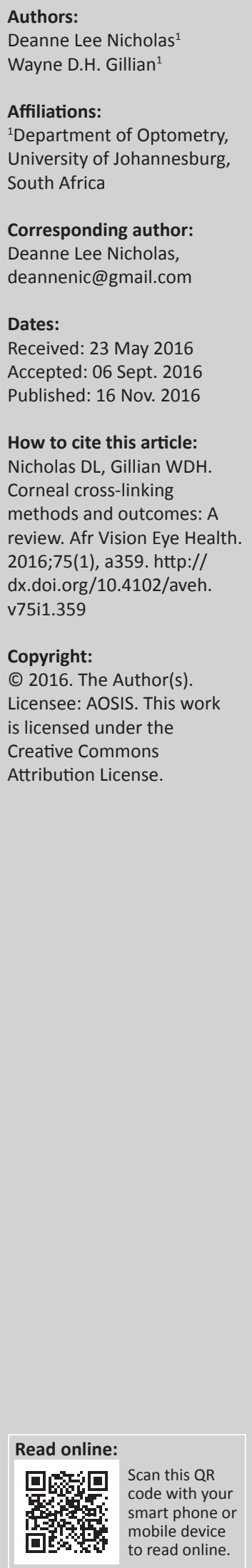

The prevalence of corneal ectasias such as keratoconus has been widely documented. Keratoconus may lead to detrimental changes in visual acuity, which can often be corrected in the early stages but requires more invasive treatment as the condition progresses. Corneal cross-linking has become a treatment method of choice in early keratoconic patients and is used to stabilise the condition and prevent further progression of the disease. The principle behind this procedure is the creation of additional bonds within the corneal structure in order to enhance its mechanical properties and thereafter halt the progression of the condition. There are disagreements within the literature as to how these procedures can be performed, and there are various alternative methods. It can be concluded that corneal cross-linking is an effective treatment method for keratoconus and has been shown to produce various beneficial effects in terms of ocular structure and function.

\section{Introduction}

Keratoconus, which is a degenerative collagen disorder, affects approximately 1 in 2000 individuals in the general population..$^{1,2}$ This corneal ectasia usually presents at late puberty and is shown to progress for approximately 10-20 years. ${ }^{3}$ Keratoconus results in changes within the ordered distribution of collagen fibrils in the cornea, leading to loss of stiffness in the stromal structure. ${ }^{1}$ The alterations occurring within the corneas of keratoconic patients lead to detrimental changes in vision as a result of increased myopia and irregular astigmatism. ${ }^{4}$ Keratoconus may be recognised by the para-central corneal thinning, the presence of Vogt's striae as well as a possible Fleischer's ring seen using a slit lamp biomicroscope, and patients with this condition may complain of ghosting and polyopia. ${ }^{3}$ The exact aetiology of keratoconus has not yet been determined; however, genetic, biochemical, environmental as well as physical factors have been suggested as causative factors in this condition. ${ }^{3}$ Because of the degenerative nature of this corneal ectasia, halting of the progression of keratoconus has become vitally important, with one of the prominent procedures, called corneal cross-linking, utilised to do so.

\section{Corneal cross-linking}

The term 'cross-linking' (CXL) refers to the creation of physical bonds between the collagen fibrils of the cornea to stabilise the corneal ectasia and prevent its further progression. The CXL procedure is employed to induce the production of cross-links through the use of riboflavin which absorbs ultraviolet A (UVA) radiation. ${ }^{3,5}$ The CXL procedure was first applied in 1998 and riboflavin was used as the substance of choice because of its absorption ability over a wide range of light spectra. ${ }^{6}$ Riboflavin has an alkylisoalloxazine structure which allows for absorption in the UVA range and is safe for systemic consumption. ${ }^{6}$ Riboflavin acts as a mediator to enhance the mechanical and biochemical stability of the cornea. ${ }^{7}$

The second component necessary for the CXL procedure is UV light, which has an absorption peak for riboflavin of $370 \mathrm{~nm}$ needed for effective cross-links to be formed; this also prevents damage to various other ocular structures. ${ }^{8}$ Riboflavin, following the absorption of UVA, acts as a photosensitiser, resulting in the production of free radicals otherwise known as oxygen singlets. ${ }^{3}$ The oxygen singlets activate the natural lysyl oxidase pathway which is thought to induce the production of cross-links. ${ }^{3}$ In addition to the lysyl oxidase pathway, the creation of cross-links is thought to occur via another process, namely, imidazolone production, which attaches itself to various other molecules, thereby creating covalent bonds. ${ }^{3}$ These covalent bonds trigger endogenous populations of carbonyl groups to form cross-links, causing degradation of the riboflavin molecule itself at the same time. ${ }^{3}$

\section{Indications for cross-linking}

The CXL procedure is generally indicated in cases of progressive keratectasia such as keratoconus. ${ }^{23,9} \mathrm{CXL}$ is the only method shown to have slowed down the progression of keratoconus. One of the first indications for the CXL procedure was for the treatment of corneal 
ulceration where the corneal tissue becomes more resistant to enzymatic digestion because of the CXL process. ${ }^{3}$ In various cases, the need for keratoplasty was avoided using this procedure. ${ }^{3,10}$ CXL has also been indicated for conditions such as bullous keratopathy, with some studies showing its efficacy in the hydration behaviour of the corneal stroma following this procedure. ${ }^{3}$ In cases of certain patients who would like to undergo laser refractive surgery, CXL is sometimes indicated because of the corneal thickness characteristics of the patient. ${ }^{9}$

\section{Types of cross-linking procedures}

CXL procedures can be divided into two broad categories: epi-on and epi-off procedures. Of great importance when considering each of the two procedures is the central corneal thickness, which should exceed $400 \mu \mathrm{m} .{ }^{3,5}$ In the standard epi-off procedure, following the instillation of topical anaesthesia, a central region of approximately $8 \mathrm{~mm}-9 \mathrm{~mm}$ of the corneal epithelium is debrided in order to enhance the absorption of the riboflavin solution. ${ }^{3}$ The epi-on procedure differs from the epi-off in that there is no removal of epithelial tissue in this process. ${ }^{11}$ The epi-off procedure results in a significant amount of pain and severe discomfort which occurs for a few days postoperatively. On the other hand, the epi-on procedure allows for increased patient comfort as there is no removal of epithelial cells prior to riboflavin installation. ${ }^{5,11}$ Another advantage of the epi-on procedure is the lower risk of infection as the epithelial cells undergo less disruption and therefore rapid visual recovery. ${ }^{12}$

Various studies have been conducted to determine the effects of each of these CXL procedures. One of the major concerns is that the epi-on technique may not allow sufficient riboflavin penetration because of the intact epithelium. The lack of disruption of epithelial cells could block approximately $20 \%$ of the UVA radiation from reaching the stroma. ${ }^{13}$ Soeters et al. found that the stabilisation effect of CXL in terms of keratometry readings was shown to be less effective with the epi-on technique. ${ }^{11}$ The readings taken using the epi-on procedure remained stable, whilst the epi-off procedure showed flattening of the keratometry readings over a 3-month postoperative period. ${ }^{11}$ However, Kocak et al. demonstrated a statistically significant improvement and stabilisation of the maximum keratometry readings in the epi-off group; this finding was absent for the epi-on group who showed deterioration of the keratometry mires following the CXL procedure. ${ }^{14}$

\section{Cross-linking combined with other procedures}

The benefits of the CXL procedure have been widely documented in the literature. However, potentiated effects have been documented when combining CXL with other refractive procedures. Although the CXL procedure may halt the progression of the corneal ectasia, patients are often left with insufficient visual acuity. Photorefractive keratectomy (PRK) was one of the first procedures to be used in combination with CXL, where the CXL halts the progression of keratoconus and PRK aids in remodelling the shape of the cornea, therefore addressing the presence of irregular astigmatism present in this condition. ${ }^{8}$ The same can be said of phototherapeutic keratectomy (PTK), where the PTK procedure aids in compensating for corneal irregularities; however, this procedure may result in additional complications as deeper corneal damage may occur as a result. ${ }^{8}$ Shetty et al. suggested the introduction of topography-guided PRK to reduce the steepness of the anterior cornea followed by the CXL procedure in order to halt the progression of keratoconus. ${ }^{1}$ The study by Kanellopoulos and Binder also advocated the use of transepithelial PRK prior to the CXL procedure and found that in the majority of cases, the PRK procedure resulted in stabilisation or flattening of the topography results and thereafter the progression of keratoconus could be managed using the CXL procedure. ${ }^{15}$

\section{Long-term results}

Various studies have been conducted where the long-term effects and benefits of the CXL procedure have been investigated. CXL may lead to various biochemical changes in corneas with progressive corneal ectasias, such as an increase in rigidity, an increase in the collagen fibre diameter, resistance to enzymatic digestion as well as resistance to hydration. ${ }^{16}$ A 24-month follow-up was performed by De Bernardo et al. on 55 patients treated with CXL, where parameters measured included best corrected visual acuity (BCVA), measurements of the corneal thinnest point, keratometry readings of the cone apex, corneal volume and axial length. ${ }^{17}$ The results of this study revealed a statistically significant improvement in the BCVA and a significant decrease (thinning) in the corneal thinnest point, the keratometry readings (Kmax values measured in dioptres) as well as the corneal volume indicating a disappearance of oedema in the corneal stroma. ${ }^{17}$ When measuring the axial length, results showed a statistically significant increase in this value which was attributed to the stabilising effect occurring in the anterior chamber depth and volume. ${ }^{17}$ Nicula et al. investigated the keratometric, refractive as well as functional changes that occurred 3 years after CXL for progressive keratoconus. ${ }^{4}$ The 3 -year follow-up yielded the following results: a statistically significant reduction in keratometric values of approximately $2.00 \mathrm{D}$, a statistically significant reduction in the spherical equivalent of $1.14 \mathrm{D}$ and an improvement in visual acuity by 1-3 lines in the majority of cases. ${ }^{4}$ Another 3-year follow-up study was performed by Khan et al. on 66 patients. ${ }^{18}$ The 3-year follow-up revealed an improvement of BCVA in $56.3 \%$ of cases, and the BCVA remained the same in $40.8 \%$ of cases. ${ }^{18}$ The spherical equivalent was shown to decrease in $33.8 \%$ of cases, whilst this remained the same in $60.6 \%$ cases and the progression of keratoconus was halted in approximately $95.8 \%$ cases. $^{18}$

A 4-year follow-up study was conducted by Vinciguerra et al. where the changes in various age groups were investigated in 301 patients following CXL for progressive keratoconus. ${ }^{2}$ 
In the paediatric population, significant improvement in BCVA was observed at 6 and 12 months postoperatively; however, this was not statistically significant at any followup visits thereafter. ${ }^{2}$ In the 18- to 29 -year-old population, the BCVA showed significant improvement up to 36 months postoperatively with no significant improvement in the spherical equivalent. ${ }^{2}$ The population groups of 30-39 years and over 40 years showed similar results with an increase in BCVA up to 36 months postoperatively with a significant improvement in the spherical equivalent at 24 months. $^{2}$

A study by Poli et al. followed 25 patients over a 6-year period measuring components such as central corneal thickness, intraocular pressure and endothelial cell density, amongst others. ${ }^{19}$ The central corneal thickness was shown to reduce significantly at every postoperative time interval ranging from 1 month to 6 years, and intraocular pressure remained stable for the first three postoperative years but was seen to increase significantly thereafter. ${ }^{19}$ Endothelial cell density did not reveal any significant changes at any postoperative point of their study. ${ }^{19}$ The conclusions of this study showed that stabilisation of the corneal ectasia without improvement in BCVA was present in $67 \%$ of cases, there was improvement in BCVA in $22 \%$ and the progression of the condition continued in $11 \%$ of these patients. ${ }^{19}$

\section{Accelerated cross-linking}

The general protocol for the CXL procedure is UV light at an intensity of $3 \mathrm{~mW} / \mathrm{cm}^{2}$ for a period of approximately $30 \mathrm{~min} \cdot{ }^{20}$ Accelerated protocols to the CXL procedure have been suggested by various studies where the treatment time is decreased whilst the intensity of the UV exposure is increased. The use of $7 \mathrm{~mW} / \mathrm{cm}^{2}$ as an intensity for approximately $15 \mathrm{~min}$ has been suggested with an assumption that sufficient cross-links will be created in order to halt the progression of keratoconus. ${ }^{8}$ Other advantages of accelerated CXL procedures include avoiding excess dehydration of the cornea and corneal thinning which may occur during the standard procedure.

The study by Hashemi et al. investigated the use of an accelerated protocol in the treatment of keratoconus. An accelerated protocol involved an intensity of $18 \mathrm{~mW} / \mathrm{cm}^{2}$ for a period of $5 \mathrm{~min}$ and this was compared with the standard protocol..$^{20}$ The visual acuity results revealed that there was no statistically significant difference between the uncorrected or the corrected distance visual acuity of the two different groups. ${ }^{20}$ In terms of keratometry readings, no significant differences could be found between the standard and the accelerated protocols. ${ }^{20}$ Sherif also conducted a comparative study where an accelerated protocol $\left(30 \mathrm{~mW} / \mathrm{cm}^{2}\right.$ for $4 \mathrm{~min}$ and $20 \mathrm{~s}$ ) was paralleled with the standard protocol. ${ }^{21}$ The results of this study found that both groups displayed a statistically significant improvement in BCVA and there was a reduction (flattening effect) of the maximum keratometry readings in both subject groups. ${ }^{21}$ In terms of complications, the transient haze experienced by the subjects treated in this study was shown to be $20 \%$ less in the accelerated group compared to the standard group. ${ }^{21}$
A 2-year follow-up study was performed by Shetty et al. where 18 paediatric patients were treated with accelerated CXL ( $9 \mathrm{~mW} / \mathrm{cm}^{2}$ at a wavelength of $365 \mathrm{~nm}$ for $10 \mathrm{~min}$ ) in order to determine long-term results. ${ }^{22}$ The results of this study revealed a statistically significant improvement in the unaided distance visual acuity, the BCVA, the cylindrical refraction as well as the keratometry readings taken 2 years following the treatment. ${ }^{22}$ The only factors in this study which did not display a statistically significant difference were the spherical refraction and the spherical equivalent. ${ }^{22}$ Shetty et al. advocate the use of accelerated CXL especially for paediatric patients as there is less discomfort with satisfactory results similar to those of the standard CXL protocol. $^{22}$

\section{Conclusion}

CXL has become widely utilised as an effective method to slow down the progression of corneal ectasias such as keratoconus. This procedure also slows down the need for a corneal graft, reduces the risk of scarring and is easy to perform and the riboflavin needed is relatively easy to access. Improvements in BCVA have been demonstrated by various studies as well as the success rates in terms of stabilising the structure of the cornea in order to prevent further damage.

\section{Acknowledgements Competing interests}

The authors declare that they have no financial or personal relationships which may have inappropriately influenced them in writing this article.

\section{Authors' contributions}

D.L.N. and W.D.H.G. were responsible for the research collection. D.L.N. was responsible for writing the research article under the supervision of W.D.H.G.

\section{References}

1. Shetty R, Nuijts R, Nicholson M, et al. Cone location-dependent outcomes after combined topography-guided photorefractive keratectomy and collagen crosslinking. Am J Ophthalmol. 2015;159(3):419-425. http://dx.doi.org/10.1016/j. ajo.2014.11.020

2. Vinciguerra R, Romano MR, Camesasca $\mathrm{FI}$, et al. Corneal cross-linking as a treatment for keratoconus. Ophthalmology. 2013;120(5):908-916. http://dx.doi. org/10.1016/j.ophtha.2012.10.023

3. O'Brart DPS. Corneal collagen cross-linking: A review. J Optom. 2014:7(3):113-124. http://dx.doi.org/10.1016/j.optom.2013.12.001

4. Nicula C, Nicula D, Popescu R, Saplonţai-Pop A. Corneal collagen cross-linking in keratoconus - Long-term prospective study. J Fr Ophtalmol. 2015:38(3):199-205. http://dx.doi.org/10.1016/j.jfo.2014.10.007

5. Kymionis GD, Portaliou DM, Diakonis VF, et al. Corneal collagen cross-linking with riboflavin and ultra-violet-A irradiation in patients with thin corneas. Am J Ophthalmol. 2012:153(1):24-28. http://dx.doi.org/10.1016/j.ajo.2011.05.036

6. Shetty R, Pahuja NK, Nuijts RMMA, et al. Current protocols of corneal collagen cross-linking: visual, refractive, and tomographic outcomes. Am J Ophthalmol. 2015;160(2):243-249. http://dx.doi.org/10.1016/j.ajo.2015.05.019

7. Spoerl E, Mrochen M, Sliney D, Trokel S, Seiler T. Safety of UVA-riboflavin crosslinking of the cornea. Cornea. 2007;26(4):385-389. http://dx.doi.org/10.1097/ ICO.0b013e3180334f78

8. Randleman JB, Khandelwal SS, Hafezi F. Corneal cross-linking. Surv Ophthalmol. 2015:60(6):509-523. http://dx.doi.org/10.1016/j.survophthal.2015.04.002

9. Dahl BJ, Spotts E, Truong JQ. Corneal collagen cross-linking: an introduction and literature review. Optometry. 2012;83(1):33-42. http://dx.doi.org/10.1016/j. optm.2011.09.011 
10. Said DG, Elalfy MS, Gatzioufas Z, et al. Collagen cross-linking with photoactivated riboflavin (PACK-CXL) for the treatment of advanced infectious keratitis with riboflavin (PACK-CXL) or the treatment of advanced infectious keratitis with
corneal melting. Ophthalmology. 2014;121(7):1377-1382. http://dx.doi. corneal melting. Ophthalmology.

11. Soeters N, Wisse RPL, Godefrooij DA, Imhof SM, Tahzib NG. Transepithelial versus epithelium-off corneal cross-linking for the treatment of progressive keratoconus: A randomized controlled trial. Am J Ophthalmol. 2015;159(5):821-828. http:// dx.doi.org/10.1016/j.ajo.2015.02.005

12. Jeng BH. Advances in medical and surgical cornea: From diagnosis to procedure. Berlin: Springer; 2014.

13. Mohammadpour $M$, Hahemi $H$, Jabbarvand $M$. Technique of simultaneous femtosecond laser assisted Myoring implantation and accelerated intrastromal collagen cross-linking for management of progressive keratoconus: A nove technique. Cont Lens Anterior Eye. 2015;39(1):1-6.

14. Kocak I, Aydin A, Kaya F, Koc H. Comparison of transepithelial corneal collagen crosslinking with epithelium-off crosslinking in progressive keratoconus. J Fr Ophtalmol. 2014;37(5):371-376. http://dx.doi.org/10.1016/j.jfo.2013.11.012

15. Kanellopoulos AJ, Binder PS. Management of corneal ectasia after LASIK with combined, same-day, topography-guided partial transepithelial PRK and collagen cross-linking: The Athens Protocol. J Refract Surg. 2011;27(5):323-331. http:// dx.doi.org/10.3928/1081597X-20101105-01
16. Barbara A. Textbook on keratoconus: New insights. New Delhi: JP Medical Ltd; 2011.

17. De Bernardo M, Capasso L, Lanza M, et al. Long-term results of corneal crosslinking for progressive keratoconus. J Optom. 2015;8(3):180-186. http://dx.doi. org/10.1016/j.optom.2014.05.006

18. Khan WA, Zaheer N, Khan S. Corneal collagen cross-linking for keratoconus: Results of 3-year follow-up in Pakistani population. Can J Ophthalmol. 2015;50(2):143-150. http://dx.doi.org/10.1016/j.jcjo.2014.11.003

19. Poli M, Lefevre A, Auxenfans C, Burillon C. Corneal collagen cross-linking for the treatment of progressive corneal ectasia: 6-year prospective outcome in a French population. Am J Ophthalmol. 2015:160(4):654-662. http://dx.doi.org/10.1016/j. ajo.2015.06.027

20. Hashemi H, Miraftab M, Seyedian MA, et al. Long-term results of an accelerated corneal cross-linking protocol $\left(18 \mathrm{~mW} / \mathrm{cm}^{2}\right)$ for the treatment of progressive keratoconus. Am Ophthalmol. 2015;160(6):1-7. http://dx.doi.org/10.1016/j.ajo.2015.08.027

21. Sherif AM. Accelerated versus conventional corneal collagen cross-linking in the treatment of mild keratoconus: A comparative study. Clin Ophthalmol. 2014;8:1435-1440. http://dx.doi.org/10.2147/OPTH.S59840

22. Shetty R, Nagaraja H, Jayadev C, Pahuja NK, Kummelil MK, Nuijts RMMA. Accelerated corneal collagen cross-linking in paediatric patients: Two-year followup results. BioMed Res 2014:1-5. 